\title{
Analysis of the Transformer Exceeding DC Resistance Unbalance Rate
}

\author{
Xu Jianguo, Pei Yujie, Wang Qinghao, Zhang Xiaoshu, Li Na, Li Bo, Liu Xiao, \\ Huang Dongwei, Su Yang, Mou Ying, Jin Jing \\ Fushun Power Supply Company, Liaoning Electric Power Company Limited, State Grid, China, \\ fushunpowersupply@163.com
}

Keywords: DC resistance; unbalance rate; exceed; transformer

\begin{abstract}
Pointed out that the lead wire resistance difference, quality, lead wire connection is not tight, tap switch bad contact, tap switch finger refers to a shift of the position pointer and winding broken strands is caused by the main reason of DC resistance unbalance rate exceed in transformer, and combined with the actual situation, proposed the corresponding solution measures.
\end{abstract}

\section{Introduction}

The DC resistance test is the necessary test items of the commissioning test and the preventive test for the power transformer, is one of the effective methods to judge the transformer whether there is a fault. Therefore, researching the DC resistance unbalance rate exceed in the transformer plays a very important role. The lead wire structure of the medium and small transformer is as shown in Figure 1, from Figure 1, the winding wire length are different, therefore, the DC resistance of each phase winding is different ( $\mathrm{Rab} \approx \mathrm{Rbc}<\mathrm{Rca}$ ), leading to the unbalance rate exceed[1-3]. In March 2002, in Liaoning Shihua University, doing the commissioning test for the SL7-315/10 type transformer of the box type substation, the factory values and the measured values of the DC resistance are exceeded in the low voltage winding, the data are shown in Table 1.

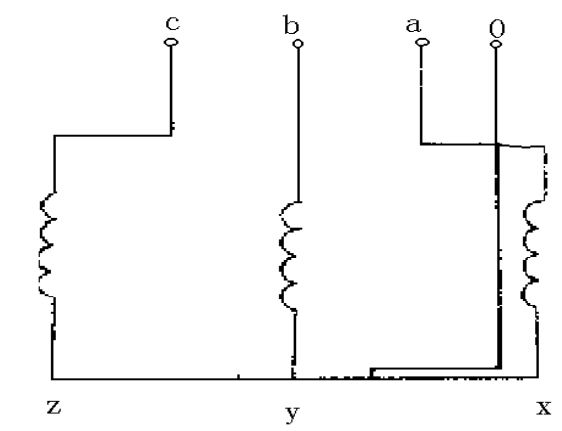

Figure 1 Transformer secondary lead wire structure diagram

Table 1 Transformer secondary DC resistance and unbalance rate

\begin{tabular}{|c|c|c|c|c|}
\hline & \multicolumn{3}{|c|}{$\mathrm{DC}$ resistance $/ \Omega$} & \multirow{2}{*}{$\begin{array}{c}\text { unbalance rate } / \% \\
\text { (phase) }\end{array}$} \\
\hline & $a_{0}$ & $\mathrm{~b}_{0}$ & $\mathrm{c}_{0}$ & \\
\hline the factory values & 0.002098 & 0.002034 & 0.002135 & 4.86 \\
\hline the measured values & 0.002036 & 0.001992 & 0.002091 & 4.85 \\
\hline
\end{tabular}

From Table 1, because of the influence of the lead wire will cause unbalance rate exceed in the transformer winding. Combined with the report data of the factory test and according to the actual experience to judge the effect of lead wire resistance, the DC resistance of the transformer although exceed (the rules $<4 \%$ ), but it can be put into operation. The transformer has been put into operation more than 2 years, without abnormalities. For the three-phase winding DC resistance are very similar in the transformer, both a and c phase winding DC resistance has been the biggest 
influence of the lead wire, therefore, the unbalance rate easily to exceed[4, 5].

\section{Lead wire loose}

Testing proved that the connection between the lead wire and the bushing guide bar or the tap switch is not tight, will lead to the DC resistance unbalance rate exceed in the transformer.

In a SJL - 1000/10 type distribution transformer of a $\mathrm{Fu}$ Shun factory, when doing the preventive test, found that the low voltage DC resistance exceed badly, the test results are as shown in Table 2. From Table 2, the transformer DC resistance unbalance rate is greater than 4\%, suspected that the winding had a problem. After inspection for lifting the core, found that the soft copper heating and discoloration of the connection joint between the $\mathrm{C}$ phase low voltage winding and the bushing conductive copper bolt, the fastening nut of the connection joint is loose. After remove the oxide layer and tightly lock the fastening nut, then measure the unbalanced rate to meet the requirements.

In a SFSL1-10000 / 66 type step-down transformer of a Fu Shun factory, when doing the commissioning test, found that the medium voltage winding DC resistance unbalance rate exceed (the results are as shown in Table 3).

Table 2 Transformer secondary DC resistances and unbalance rate

\begin{tabular}{|c|c|c|c|c|}
\hline \multirow{2}{*}{ Test time } & \multicolumn{3}{|c|}{ DC resistance $/ \Omega$} & \multirow{2}{*}{ - unbalance rate $/ \%$} \\
\hline & $a_{O}$ & $b_{\mathrm{O}}$ & $\mathrm{c}_{\mathrm{O}}$ & \\
\hline the preventive test & 0.001072 & 0.001073 & 0.001495 & 39. 46 \\
\hline treatment & 0.001072 & 0.001073 & 0.001081 & 0.84 \\
\hline
\end{tabular}

Table 3 The commissioning test results of transformer primary DC resistance

\begin{tabular}{|c|c|c|c|c|}
\hline \multirow{2}{*}{ Tap position } & \multicolumn{3}{|c|}{$\mathrm{DC}$ resistance $/ \Omega$} & \multirow{2}{*}{ unbalance rate $/ \%$} \\
\hline & Ao & Bo & $\mathrm{Co}$ & \\
\hline IV & 0.316 & 0.385 & 0. 317 & 20.3 \\
\hline V & 0. 308 & 0.346 & 0. 307 & 12.18 \\
\hline
\end{tabular}

From Table 3, in transformer the medium voltage DC resistance unbalance rate is more than $2 \%$ (DL/T 596-596 rule value). Inspection for lifting the cover, found that in the B phase medium voltage winding,the connection of the sixth tap lead wire with tap switch conductive column is loose.

In a $10000 \mathrm{kVA}, 60 \mathrm{kV}$ on-load voltage regulating transformer of a substation, when doing the preventive test, found that the DC resistance is unqualified, the results are as shown in Table 4.

From Table 4, in the three tap position, the B phase DC resistance is more about $7 \%$ than the other two phase. Inspection for outage and found that the B phase through the bushing lead wire's nose to contact with the cap is not tight.

Remove that connection is not tight should take the following measures. Improve the quality of installation and maintenance, inspection for whether the connection parts are connected well. In the operation, it can comprehensive judge the analysis results by chromatography, timely inspect the bad position. In the operation, it can use infrared test, timely detection of bad contact site, early treatment as soon as possible. 
Table 4 the preventive test results of transformer primary DC resistance

\begin{tabular}{|c|c|c|c|c|}
\hline \multirow{2}{*}{ Tap position } & \multicolumn{3}{|c|}{ DC resistance $/ \Omega$} & \multirow{2}{*}{ unbalance rate $/ \%$} \\
\hline & $\mathrm{A}_{0}$ & $\mathrm{~B}_{0}$ & $\mathrm{C}_{0}$ & \\
\hline VII & 1. 140 & 1. 217 & 1. 139 & 6.7 \\
\hline VIII & 1. 118 & 1. 198 & 1. 116 & 7. 1 \\
\hline IX & 1. 139 & 1. 219 & 1. 137 & 7. 0 \\
\hline
\end{tabular}

\section{Tap switch bad contact}

For load and no-load, tap switch bad contact is the largest number of various kinds defects in the main transformer, accounting for about $40 \%$. Bring a great threat to the safe operation of the transformer.

In a SFSLB1 - 20000 / 110 type main transformer of the East China Bureau, in the preventive test the three-phase DC resistance is balanceable.But after 8 months of operation, in the $110 \mathrm{kV}$ side, the $\mathrm{C}$ phase bushing spout oil, the temperature to $84 \mathrm{C}$.Through chromatographic analysis to consider that the transformer exist internal thermal fault, the hottest temperature to $150 \sim 300$ $\mathrm{C}$, analyzed that the conductive loop caused by bad contact. In the DC resistance test, the operation of medium voltage's tap position (IV) A 0 is $0.286 \Omega, \mathrm{B} 0$ is $0.281 \Omega, \mathrm{C} 0$ is $0.35 \Omega$, the unbalance rate is $24.55 \%$, the test results of the other parts is normal. So narrow the range of defects to the medium voltage c phase winding, lead wire $\rightarrow$ tap switch $\rightarrow$ bushing. Inspection for lifting the core founded that the moving contact and the fixed contact of the tap switch IV is bad contact in the medium voltage $\mathrm{c}$ phase, And it has the phenomenon of the overheating, discoloration and burning. After replacing the tap switch, it operates well.

The direct reason that tap switch bad contact is the pressure of the contact point was not enough, the contact surface of the coating materials is easy to oxidation, the most fundamental reason is the structural design is unreasonable, and there is no effective measures are taken. There are several ways to improve the bad contact.

\section{Tap switch finger refers to a shift of the position pointer}

Tap switch finger refers to a shift of the position pointer will cause the transformer winding DC resistance unbalance rate exceed. In a SFPS7-120000/220 type transformer of a substation, the tap switch connecting with the transformer winding is as shown in Figure 2.

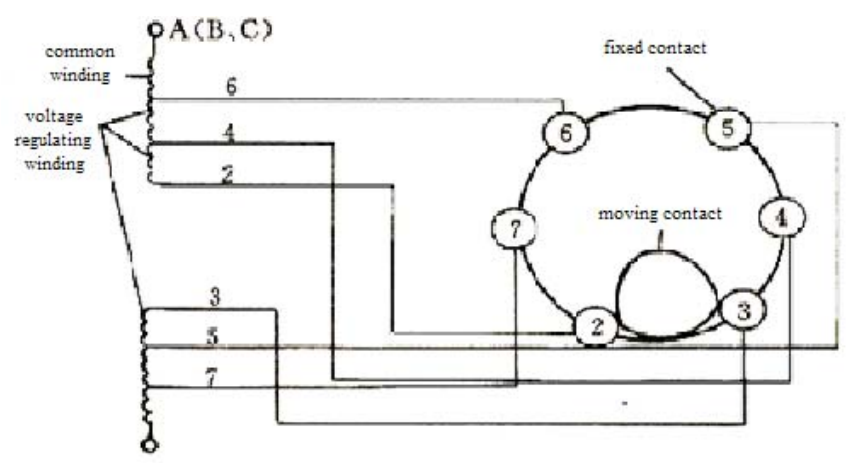

Figure 2 The tap switch connecting with the transformer winding

In the transformer insulation test, found that the high voltage winding DC resistance are serious imbalance, and the B phase DC resistance has a problem, since I - V gear value presented by big small - less - more irregular arrangement, the test results are as shown in Table 5.Through analysis to consider that the B phase gear disorder, decided to inspection for cover lifting, when hanging the 
cover lifting, the maintenance personnel will put in the switch connecting rod, it has no check nails and rotates freely. Then according to the correct position of the B phase adjust the tap switch, make the check nails keep off the block gear, measured the three-phase DC resistance are balance and meet the requirement of limit value. Test results are as shown in Table 6.

Table 5 Test results for the high voltage winding DC resistance

\begin{tabular}{cccccc}
\hline Tap gear & I & II & III & IV & V \\
\hline fixed contact & $2-3$ & $3-4$ & $4-5$ & $5-6$ & $6-7$ \\
A- $0 / \Omega$ & 0.6583 & 0.6308 & 0.6113 & 0.5920 & 0.5721 \\
B- $0 / \Omega$ & 0.6102 & 0.5921 & 0.5723 & 0.6104 & 0.6584 \\
C- $0 / \Omega$ & 0.6581 & 0.6308 & 0.6102 & 0.5919 & 0.5722 \\
unbalance rate $/ \%$ & 7.40 & 6.26 & 6.50 & 3.10 & 14.30 \\
\hline
\end{tabular}

Table 6 Test results for the high voltage winding DC resistance

\begin{tabular}{|c|c|c|c|c|c|}
\hline tap switch position & I & II & III & IV & V \\
\hline $\mathrm{A}-0 / \Omega$ & 0.6436 & 0.6226 & 0.6017 & 0.5829 & 0.5600 \\
\hline $\mathrm{B}-0 / \Omega$ & 0.6445 & 0.6232 & 0.6015 & 0.5813 & 0.5604 \\
\hline $\mathrm{C}-0 / \Omega$ & 0.6448 & 0.6233 & 0.6019 & 0.5816 & 0.5608 \\
\hline unbalance rate $/ \%$ & 0.14 & 0.11 & 0.07 & 0.27 & 0.14 \\
\hline
\end{tabular}

In order to eliminate tap switch finger refers to a shift of the position pointer caused by the DC resistance unbalance rate exceed, before the factory it should do the tap switch test, check whether the tap switch position is right. After maintenance the tap switch, it should also check.

\section{Conclusion}

The main reasons that the transformer DC resistance exceed are the lead wire connection is not tight, tap switch bad contact, tap switch finger refers to a shift of the position pointer and winding broken strands.

\section{Reference}

[1] Dong Qiguo. Power Transformer Taults And The Diagnosis. China Power Press,2000.

[2] Chen Huagang. The Preventive Test Electrical Equipment Technical Question and Answer. Water Conservancy Electric Power Press,1998.

[3] Chen Huagang. Electrical equipment preventive test method. Water Conservancy Electric Power Press, 1999.

[4] Han Aizhi. The simple method of judging transformer winding deformation, transformer, 2003]

[5] Chen Tianxiang, Wang Yinzhong. Electrical Test. China power press, 2010 